\title{
Figure S2
}

A

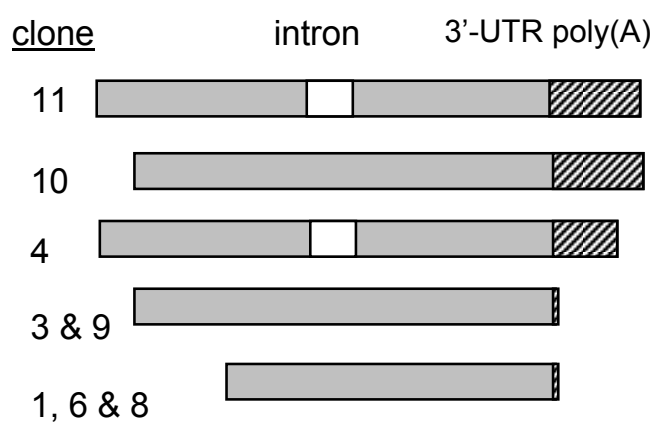

B

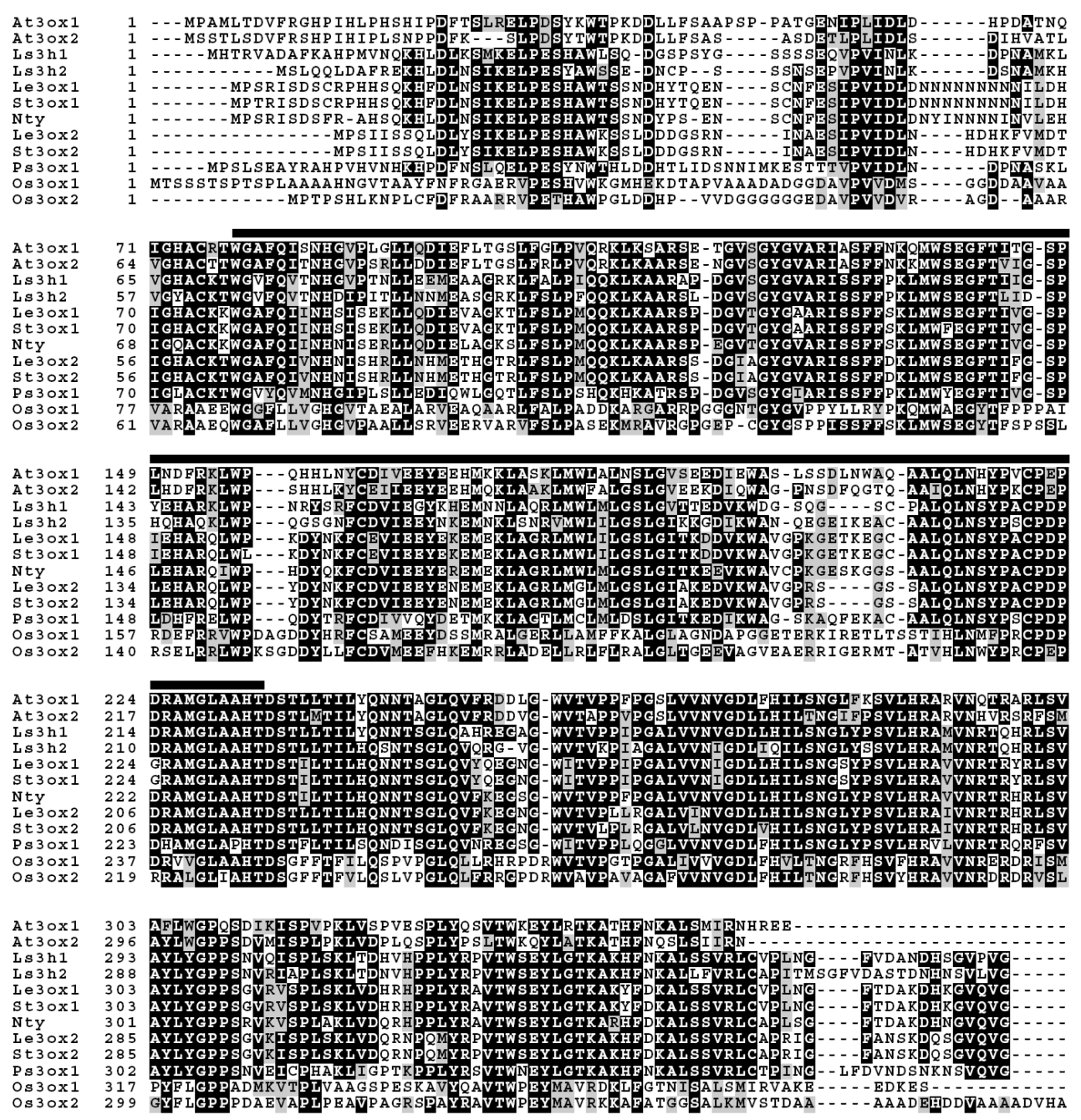

Figure S2. Structure of StGA3ox2 clones isolated from potato. (A) Schematic representation of the different clones corresponding to StGA3ox2 mRNA. An unspliced intron is present in clones 11 and 4 . Multiple poly-adenylation sites are used during transcription, with a poly-A tail added directly after the stop codon in clones 3 \& 9, or clones 1,6 \& 8. (B) Comparison of potato StGA3ox1 and StGA3ox2 amino acid sequences with other GA 3-oxidases from Arabidopsis (At3ox1 NM 101424 and At3ox2 NM 106683), pea (Ps3ox1 AF010167), lettuce (Ls3h1 AB012205 and Ls3h2 AB012206), tomato (Le3ox1 Le3ox2 $\approx$ SI3ox2 AB010992), tobacco (Nty AB032198) and rice (Os3ox1 AB054084 and Os3ox2 AB056519) genes. . Black boxes indicate identical residues, and gray boxes indicate conserved residues. Dashes indicate gaps in the sequences. Sequence corresponding to the B3ox fragment is shown on top of the alignment with a thick black line. 